\title{
Generalized Analytic and Quasi-Analytic Vectors
}

\author{
by \\ Jan RUSINEK \\ Presented by Jan KISYŃSKI
}

Summary. For every sequence $\left(a_{n}\right)$ of positive real numbers and an operator acting in a Banach space, we introduce the families of $\left(a_{n}\right)$-analytic and $\left(a_{n}\right)$-quasi-analytic vectors. We prove various properties of these families.

Introduction. Let $E$ be a Banach space, and $A$ an operator (bounded or unbounded) acting in $E$. Various sets of vectors, members of $E$, can be associated with $A$. The simplest examples include the domain $D(A)$ of $A$, and the set

$$
C^{\infty}(A)=\bigcap_{n=1}^{\infty} D\left(A^{n}\right)
$$

of $C^{\infty}$-vectors for $A$.

If a given operator has some geometric properties (for example, is symmetric acting in Hilbert space) and has a sufficiently large (say, dense) set of vectors of a special class, then the operator often has useful properties: it is essentially self-adjoint or generates a strongly continuous group or semigroup.

Important "classical" classes of vectors are those of analytic vectors, quasi-analytic vectors, semi-analytic vectors, and Stieltjes vectors.

In this paper we shall consider the following more general sets of vectors.

Definition 1. Let $\left(c_{n}\right)$ be a sequence of strictly positive numbers. An element $x \in C^{\infty}(A)$ belongs to $\mathcal{A}_{\left(c_{n}\right)}(A)$ if

$$
\sum_{n=1}^{\infty} \frac{\left\|A^{n} x\right\|}{c_{n}} t^{n}<\infty \quad \text { for some } t>0 .
$$

2000 Mathematics Subject Classification: 40A05, 40A20, 47B25, 47B37, 47B65.

Key words and phrases: analytic vector, essentially self-adjoint operator. 
Definition 2. Let $\left(b_{n}\right)$ be a sequence of strictly positive numbers. An element $x \in C^{\infty}(A)$ belongs to $\mathcal{Q}_{\left(b_{n}\right)}(A)$ if

$$
\sum_{n=1}^{\infty}\left\|A^{n} x\right\|^{-1 / b_{n}}=\infty .
$$

Note that the same classes $\mathcal{A}_{\left(c_{n}\right)}(A)$ can be obtained by using different sequences $\left(c_{n}\right)$. Indeed,

$$
\mathcal{A}_{\left(c_{n}\right)}(A)=\left\{x \in C^{\infty}(A): \sup _{n=1,2, \ldots} \frac{\left\|A^{n}\right\|}{c_{n}} t^{n}<\infty \text { for some } t>0\right\},
$$

which implies the following

Proposition 1. Let $\left(c_{n}\right)$ and $\left(c_{n}^{\prime}\right)$ be two sequences of positive numbers such that

$$
c_{n} \leq c_{n}^{\prime} d^{n} \quad \text { for some } d>0 \text {. }
$$

Then

$$
\mathcal{A}_{\left(c_{n}\right)}(A) \subset \mathcal{A}_{\left(c_{n}^{\prime}\right)}(A) .
$$

Proposition 1 and Stirling's formulae imply that for example $\mathcal{A}_{\left((n !)^{p}\right)}(A)$ $=\mathcal{A}_{\left(n^{p n}\right)}(A)$.

For the sets $\mathcal{Q}_{\left(b_{n}\right)}(A)$ we have the following

Proposition 2. If $0<b_{n} \leq b_{n}^{\prime}<\infty$ for every $n=1,2, \ldots$, then

$$
\mathcal{Q}_{\left(b_{n}\right)}(A) \subset \mathcal{Q}_{\left(b_{n}^{\prime}\right)}(A) \text {. }
$$

Proof. Indeed, if $\left\|A^{n} x\right\| \leq 1$ for infinitely many $n$ 's, then $x \in \mathcal{Q}_{\left(b_{n}\right)}(A) \cap$ $\mathcal{Q}_{\left(b_{n}^{\prime}\right)}(A)$. Furthermore, if $\left\|A^{n} x\right\|>1$, then $\left\|A^{n} x\right\|^{-1 / b_{n}} \leq\left\|A^{n} x\right\|^{-1 / b_{n}^{\prime}}$, so that if $\left\|A^{n} x\right\| \leq 1$ for finitely many $n$ 's only and $x \in \mathcal{Q}_{\left(b_{n}\right)}(A)$, then $x \in \mathcal{Q}_{\left(b_{n}\right)}(A)$.

For unbounded symmetric operators $A$ in a Hilbert space $H$ the denseness of $\operatorname{lin} \mathcal{A}_{\left(c_{n}\right)}(A)$ or lin $\mathcal{Q}_{\left(b_{n}\right)}(A)$ in $H$ may imply the essential self-adjointness of $A$.

If $c_{n}=n$ ! (or $\left.n^{n}\right)$ then $\mathcal{A}_{\left(c_{n}\right)}(A)$ coincides with the set of analytic vectors introduced by Nelson [4], who proved that a symmetric operator with a linearly dense set of analytic vectors is essentially self-adjoint.

In the case of $b_{n}=n$ we obtain the quasi-analytic vectors introduced by Nussbaum [5], who showed a more general result stating that a symmetric operator with a linearly dense set of quasi-analytic vectors is essentially self-adjoint.

If $c_{n}=(2 n)$ !, then $\mathcal{A}_{\left(c_{n}\right)}(A)$ coincides with the semi-analytic vectors introduced by Simon [10], who proved that a symmetric semi-bounded operator with a linearly dense set of semi-analytic vectors is essentially selfadjoint. 
If $b_{n}=2 n$, then $\mathcal{Q}_{\left(b_{n}\right)}(A)$ is equal to the set of Stieltjes vectors introduced by Nussbaum [6], who showed that a symmetric semi-bounded operator with a linearly dense set of Stieltjes vectors is essentially self-adjoint.

The following diagram displays the relationships between various classes of vectors:

\section{analytic $\subset$ quasi-analytic \\ $\cap \quad \cap$ \\ semi-analytic $\subset$ Stieltjes vectors}

The sets $\mathcal{A}_{\left(c_{n}\right)}(A)$ also play an important role for unbounded operators $A$ in Banach spaces. Let $p$ be a positive real number. If $c_{n}=n^{p n}$ or $c_{n}=(n !)^{p}$, then we obtain the space called in [1] the abstract Gevrey space of order $p$ associated with $A$; this space is denoted by $G(p)$ and called the space of p-analytic vectors in [3] and [8].

In [1] only closed operators were considered and it was proved that under some assumptions on the resolvent, a closed operator $A$ generates a strongly continuous semigroup in $G(p)$ equipped with some locally convex topology.

If $b_{n}=p n$, then we obtain the $p$-quasi-analytic vectors considered in [3] and [8]. With this terminology, analytic vectors are simply 1-analytic, semi-analytic ones are 2-analytic, quasi-analytic ones are 1-quasi-analytic, and Stieltjes vectors are 2-quasi-analytic.

When $E$ is the space of bounded continuous functions on an interval in $\mathbb{R}$ and $A=d / d x$, special cases of spaces $\mathcal{A}_{\left(c_{n}\right)}(A)$ are considered in [7], namely such that from $f \in \mathcal{A}_{\left(c_{n}\right)}(A)$ and $\left(A^{n} f\right)\left(x_{0}\right)=0$ for $n=0,1,2, \ldots$ it follows that $f(x) \equiv 0$. Such classes are called quasi-analytic.

If $c_{n}=(n !)^{p}$ and $E, A$ are as above and $p \in(1, \infty)$, then $\mathcal{A}_{\left(c_{n}\right)}(A)$ is the classical space of Gevrey functions of order $p$ (see [2]), and if $p \in(0,1]$, then $\mathcal{A}_{\left(c_{n}\right)}(A)$ is a quasi-analytic class.

$\mathcal{A}_{\left(c_{n}\right)}(A)$ vectors and $\mathcal{Q}_{\left(b_{n}\right)}(A)$ vectors. We shall show some connections between the two classes of vectors defined above. We start with the following result.

THEOREM 1. Let $\left(c_{n}\right)$ and $\left(b_{n}\right)$ be sequences of positive numbers such that for some $a>0$,

$$
b_{n} \geq \max \left(a n, \frac{\ln c_{n}}{\ln n}\right) \quad(n \in \mathbb{N}) .
$$

Then, for any operator $A$,

$$
\mathcal{A}_{\left(c_{n}\right)}(A) \subset \mathcal{Q}_{\left(b_{n}\right)}(A) .
$$


Proof. Let $x \in \mathcal{A}_{\left(c_{n}\right)}(A)$. Then

$$
\sum_{n=1}^{\infty} \frac{\left\|A^{n} x\right\|}{c_{n}} t^{n}
$$

has a positive radius of convergence equal to $1 / r$, where

$$
r=\limsup _{n \rightarrow \infty} \sqrt[n]{\left\|A^{n} x\right\| / c_{n}}<\infty .
$$

Let $M>\max (1, r)$. Then there exists $n_{0}$ such that for $n>n_{0}$,

$$
\sqrt[n]{\left\|A^{n} x\right\| / c_{n}}<M
$$

Hence

$$
\left\|A^{n} x\right\|^{-1 / b_{n}}>\frac{1}{M^{n / b_{n}}\left(c_{n}\right)^{1 / b_{n}}} .
$$

Since $b_{n} \geq a n$, we see that $M^{n / b_{n}} \leq M^{1 / a}$, and since $b_{n} \geq \ln c_{n} / \ln n$, we have $\left(c_{n}\right)^{1 / b_{n}} \leq n$. Finally,

$$
\left\|A^{n} x\right\|^{-1 / b_{n}}>1 / M^{1 / a} n
$$

which implies $\sum_{n=1}^{\infty}\left\|A^{n} x\right\|^{-1 / b_{n}}=\infty$.

As a simple corollary, we establish the horizontal inclusions in the diagram above. Indeed, it suffices to let $c_{n}=n^{n}$ and $b_{n}=n$ to obtain the upper inclusion, and $c_{n}=n^{2 n}$ and $b_{n}=2 n$ to obtain the lower inclusion.

REMARK. The condition $b_{n} \geq a n$ is a very natural one if the denseness of $\mathcal{Q}_{\left(b_{n}\right)}(A)$ for a bounded $A$ is to be ensured. Suppose that each $\left(b_{n}\right)$ is slightly less than $a n$, for example $b_{n}=n^{1-\varepsilon}$. Let $A$ be the scalar operator $A x=2 x$ and let $x$ be an arbitrary non-zero vector from $E$. Then $\left\|A^{n} x\right\|=$ $2^{n}\|x\|$, whence $\left\|A^{n} x\right\|^{-1 / b_{n}}=2^{-\left(n^{\varepsilon}\right)}\|x\|^{1-\varepsilon}$. Since $2^{n^{\varepsilon}}>n^{2}$ for large $n$, we see that the series $\sum_{n=1}^{\infty}\left\|A^{n} x\right\|^{-1 / b^{n}}$ is convergent, and consequently that $x \notin \mathcal{Q}_{\left(b_{n}\right)}(A)$.

If we let $c_{n}=n^{n}$ and $b_{n}=n$ and consider analytic vectors and quasianalytic vectors, then the expected growth of $\left(\left\|A^{n} x\right\|\right)$ is similar. The main difference is that in the latter case this growth can be much more irregular. Therefore results concerning vectors in $\mathcal{Q}_{\left(b_{n}\right)}(A)$ are in general stronger than those for $\mathcal{A}_{\left(c_{n}\right)}(A)$. This phenomenon is demonstrated by the following theorem which is the main result of this paper.

THEOREM 2. Let $\left(c_{n}\right)$ and $\left(b_{n}\right)$ be sequences of strictly positive numbers with $b_{n} \geq$ an for some $a>0$. There exists a symmetric operator $A$ acting in a Hilbert space $H$ such that the set $\mathcal{Q}_{\left(b_{n}\right)}(A)$ is dense in $H$ and the set $\mathcal{A}_{\left(c_{n}\right)}(A)$ comprises only the zero vector.

Proof. Without loss of generality we may assume that $c_{n} \geq 1$ for $n=$ $1,2, \ldots$ Let $H=l^{2}$ be the Hilbert space of all square-summable complex 
sequences. Let $e_{k}=(0, \ldots, 0,1,0, \ldots)$ (1 at the $k$ th place) be the standard basis in $H$. Let $m_{0}$ be the linear subspace of $H$ spanned by $e_{1}, e_{2}, \ldots$ Of course, $m_{0}$ is dense in $H$.

For a sequence $\left(a_{k}\right)(k=1,2, \ldots)$, let additionally $a_{0}=0$ and consider the operator $A$ defined on $m_{0}$ as follows:

$$
A e_{k}=a_{k-1} e_{k-1}+a_{k} e_{k+1} \quad \text { for } k=1,2, \ldots
$$

In matrix form, $A$ is given by the Jacobi matrix

$$
\left[\begin{array}{cccccc}
0 & a_{1} & 0 & 0 & 0 & \ldots \\
a_{1} & 0 & a_{2} & 0 & 0 & \ldots \\
0 & a_{2} & 0 & a_{3} & 0 & \ldots \\
0 & 0 & a_{3} & 0 & a_{4} & \ldots \\
\ldots \ldots & \ldots & \ldots & \ldots & \ldots
\end{array}\right]
$$

If the $a_{k}$ are real numbers, then $A$ is symmetric.

First we shall prove the following

LEMMA 1. If $a_{j}>0$ for each $j=1,2, \ldots$, then

$$
A^{n} e_{k}=\sum_{i=1}^{n+k} \alpha_{i}^{n, k} e_{i}
$$

and

(1) $\alpha_{i}^{n, k} \geq 0$

(2) $\alpha_{n+k}^{n, k}=a_{k} a_{k+1} \ldots a_{n+k-1}$,

(3) for any $q \leq n+k, \alpha_{q}^{n, k} \leq 2^{n} M^{n}$, where $M=\sup \left\{a_{i}: 1 \leq i \leq n+k\right\}$.

Proof. We proceed by induction on $n$. For $n=1$ conditions (1)-(3) result from the definition of $A$. Suppose that (1)-(3) hold for some $n$, all $k$ and all $q \leq k+n$. We have

$$
\text { (**) } \quad A^{n+1} e_{k}=A A^{n} e_{k}=A\left(\sum_{i=1}^{n+k} \alpha_{i}^{n, k} e_{i}\right)=\sum_{i=1}^{n+k} \alpha_{i}^{n, k}\left(a_{i-1} e_{i-1}+a_{i} e_{i+1}\right) \text {. }
$$

This establishes condition (1) for $n+1$.

The vector $e_{n+k+1}$ occurs in (**) only once, for $i=k+1$, with coefficient $\alpha_{n+k}^{n, k} \cdot a_{n+k}$. Hence we get (2) for $n+1$ and (3) for $q=n+k+1$.

Now let $q<n+k+1$. The vector $e_{q}$ occurs in (**) twice: for $i=q-1$ and $i=q+1$. The coefficient $\alpha_{q}^{n+1, k}$ is equal to $a_{q-1} \alpha_{q-1}^{n, k}+a_{q} \alpha_{q+1}^{n, k}$. Hence, by the inductive hypothesis, $\alpha_{q}^{n+1, k} \leq 2 M 2^{n} M^{n}=2^{n+1} M^{n+1}$.

Let now $\left(c_{n}\right)$ and $\left(b_{n}\right)$ be sequences of positive numbers with $b_{n} \geq a n$ $(a>0)$. We inductively define a Jacobi matrix of the form as in $(*)$, some 
increasing sequences $\left(k_{n}\right)$ and $\left(k_{n}^{\prime}\right)$ of natural numbers and also a sequence $\left(Q_{2 n+1}\right)$ of positive numbers.

First let $k_{1}=k_{1}^{\prime}=1, k_{2}=3, k_{2}^{\prime}=2$ and let $a_{1}=a_{2}=a_{3}=Q_{1}=$ $Q_{3}=Q_{5}=1$. Suppose that we have defined $k_{1}<k_{2}<\ldots<k_{2 n-1}<k_{2 n}$; $k_{2}^{\prime}<k_{4}^{\prime}<\ldots<k_{2 n}^{\prime} ; Q_{1}, Q_{3}, \ldots, Q_{2 n-1}$ and $a_{1}, a_{2}, \ldots, a_{k_{2 n}}$ such that for $i=0,1, \ldots, n-1$ the following three conditions are satisfied:

(a) $a_{p}=c_{p+1}(p+1)^{p+1}$ for $p=k_{2 i+1}, i=0,1, \ldots, n-1$.

(b) $a_{p}=1$ for $p \neq k_{2 i+1}, i=0,1, \ldots, n-1$.

(c) $k_{2 i+2}^{\prime}-k_{2 i+1}>\left(2 Q_{2 i+1}\right)^{1 / a}$.

Let $k_{2 n+1}=k_{2 n}+1$ and let $a_{k_{2 n+1}}=c_{p} p^{p}$ with $p=k_{2 n+1}+1$. Define $Q_{2 n+1}=\sup \left\{a_{i}: 1 \leq i \leq k_{2 n+1}\right\}$, choose $k_{2 n+2}^{\prime}$ large enough so that $k_{2 n+2}^{\prime}-k_{2 n+1}>\left(2 Q_{2 n+1}\right)^{1 / a}$, and let $k_{2 n+2}=k_{2 n+2}^{\prime}+n$. Finally, for $i=$ $k_{2 n}+2, k_{2 n}+3, \ldots, k_{2 n+2}$, let $a_{i}=1$. Directly from the construction it follows that conditions (a), (b), (c) hold for every $i=0,1, \ldots$

We now prove two lemmas.

Lemma 2. Each $x \in m_{0}$ belongs to $\mathcal{Q}_{\left(b_{n}\right)}(A)$.

Proof. Let

$$
x=\sum_{k=1}^{K} d_{k} e_{k}, \quad L=\sup \left\{\left|d_{k}\right|: k=1, \ldots, K\right\} .
$$

Let $n>K$ and $p$ be such that $k_{2 n+1}<p \leq k_{2 n+2}^{\prime}$. Then

$$
A^{p} x=\sum_{k=1}^{K} d_{k} A^{p} e_{k}
$$

By (3) and Lemma 1,

$$
\left\|A^{p} x\right\| \leq K L 2^{p} M^{p}, \quad \text { where } \quad M=\sup \left\{a_{i}: 1 \leq i \leq p+K\right\} .
$$

Since $p+K \leq k_{2 n+2}^{\prime}+K<k_{2 n+2}^{\prime}+n<k_{2 n+2}$, we see that $a_{i}=1$ for $i>k_{2 n+1}$, and $a_{i} \leq Q_{2 n+1}$ for $i \leq k_{2 n+1}$. Hence, for $k_{2 n+1}<p \leq k_{2 n+2}^{\prime}$,

$$
\left\|A^{p} x\right\|^{1 / b_{p}} \leq\left(K L 2^{p} Q_{2 n+1}^{p}\right)^{1 / b_{p}} \leq\left(K L 2^{p} Q_{2 n+1}^{p}\right)^{1 / a p}=(K L)^{1 / a p}\left(2 Q_{2 n+1}\right)^{1 / a} .
$$

Obviously, $k_{2 n+1}>n$ for each $n$. Thus, for $n>K$,

$$
\sum_{p=k_{2 n+1}+1}^{k_{2 n+2}^{\prime}}\left\|A^{p} x\right\|^{-1 / b_{p}} \geq \frac{k_{2 n+2}^{\prime}-k_{2 n+1}}{(K L)^{1 / a n}\left(2 Q_{2 n+1}\right)^{1 / a}}>\frac{1}{(K L)^{1 / a n}}=\frac{1}{\sqrt[n]{(K L)^{1 / a}}} .
$$

Since $\sqrt[n]{(K L)^{1 / a}}$ tends to 1 as $n \rightarrow \infty$, there exists $n_{0}$ such that for $n>n_{0}$ 
the above sum is greater than $1 / 2$. Therefore

$$
\begin{aligned}
\sum_{p=1}^{\infty}\left\|A^{p} x\right\|^{-1 / b_{p}} & \geq \sum_{p=k_{2 n_{0}}}^{\infty}\left\|A^{p} x\right\|^{-1 / b_{p}} \\
& \geq \sum_{n=n_{0}}^{\infty} \sum_{p=k_{2 n+1}+1}^{k_{2 n+2}^{\prime}}\left\|A^{p} x\right\|^{-1 / b_{p}}>\sum_{n=n_{0}}^{\infty} 1 / 2=\infty
\end{aligned}
$$

Lemma 3. No nonzero $x \in m_{0}$ belongs to $\mathcal{A}_{\left(c_{n}\right)}(A)$.

Proof. Let $x=\sum_{k=1}^{K} d_{k} e_{k}$, and $\left|d_{K}\right|>0$. We shall estimate $\left\|A^{p} x\right\|$ for $p=k_{2 n+1}$ with $p>K$. Since

$$
A^{p} x=\sum_{k=1}^{K} d_{k} A^{p} e_{k},
$$

it follows from Lemma 1 that

$$
A^{p} x=\sum_{k=1}^{K} d_{k} \sum_{i=1}^{p+k} \alpha_{i}^{p, k} e_{i}
$$

The vector $e_{p+K}$ occurs in this sum only once: when $k=K$ and $i=p+K$. Thus by condition (2) of Lemma 1 , the corresponding coefficient is equal to $\alpha_{p+K}^{p, K}=a_{K} a_{K+1} \ldots a_{p+K-1}$. As $K<p \leq p+K-1$, one of the factors of this product is $a_{p}=c_{p} p^{p}$. Since $c_{n} \geq 1$ for every $n$, the remaining factors are not less than 1 , and it follows that

$$
\left\|A^{p} x\right\| \geq\left|d_{K}\right| \cdot c_{p} p^{p} .
$$

Thus

$$
\sqrt[p]{\left\|A^{p} x\right\| / c_{p}} \geq \sqrt[p]{\left|d_{K}\right|} p
$$

and so

$$
\limsup _{p \rightarrow \infty} \sqrt[p]{\left\|A^{p} x\right\| / c_{p}}=\infty
$$

The theorem results immediately from the last two lemmas.

As a corollary, we obtain

COROLlary 1. For any strictly positive sequence $\left(c_{n}\right)$ there exists an essentially self-adjoint operator $A$ for which $\mathcal{A}_{\left(c_{n}\right)}(A)$ consists only of the zero vector.

Proof. An application of Theorem 2 with $b_{n}=n$ for each $n \in \mathbb{N}$ yields a symmetric operator $A$ with a linearly dense set of quasi-analytic vectors and with $\mathcal{A}_{\left(c_{n}\right)}(A)=\{0\}$. This operator is essentially self-adjoint by Nussbaum's theorem. 


\section{References}

[1] R. Beals, Semigroups and abstract Gevrey spaces, J. Funct. Anal. 10 (1972), 300-308.

[2] M. Gevrey, Sur la nature analytique des solutions des équations aux dérivées partielles, Ann. Sci. École Norm. Sup. 35 (1918), 129-189.

[3] V. I. Istrățescu, Introduction to Linear Operator Theory, Dekker, New York, 1981.

[4] E. Nelson, Analytic vectors, Ann. of Math. 70 (1959), 572-615.

[5] A. E. Nussbaum, Quasi-analytic vectors, Ark. Mat. 6 (1965), 179-191.

[6] -, A note on quasi-analytic vectors, Studia Math. 33 (1969), 305-309.

[7] W. Rudin, Real and Complex Analysis, 2nd ed., McGraw-Hill, 1974.

[8] J. Rusinek, p-Analytic and p-quasi-analytic vectors, Studia Math. 127 (1998), 233250.

[9] - Non-linearity of the set of p-quasi-analytic vectors for some essentially selfadjoint operators, Bull. Polish Acad. Sci. Math. 48 (2000), 287-292.

[10] B. Simon, The theory of semi-analytic vectors: A new proof of a theorem of Masson and McClary, Indiana Univ. Math. J. 20 (1970/71), 1145-1151.

Jan Rusinek

Cardinal Stefan Wyszyński University

Faculty of Mathematics and Natural Sciences

College of Sciences

Dewajtis 5

01-815 Warszawa, Poland

E-mail: rusinek@uksw.edu.pl 